\title{
Proximal velocity change as a determinant of space perception*
}

\author{
CLAES von HOFSTEN \\ University of Uppsala, Svartbäcksgatan 10, S-75320 Uppsala, Sweden
}

\begin{abstract}
How and to what degree does proximal velocity change determine perceived translatory motion in depth? This question was studied with a stimulus consisting of a single dot, moving in a straight horizontal path in a frontoparallel plane. Its motion corresponded to distal depth motion with constant speed. Ss reported verbally what they perceived. The results show that proximal velocity changes of this kind are, within certain limits, utilized by the visual system for the perception of translatory motion in depth. The limits were found to be determined by the absolute rate of change in proximal velocity. Further, it was found that the perceived motion track was usually bent, although all stimuli simulated depth motions along straight paths.
\end{abstract}

It is a well-established fact that spatial properties of changing proximal stimuli can evoke perceived motion in depth (Wallach \& O'Connell, 1953; Johansson, 1964; Börjesson \& von Hofsten, 1972, 1973). An additional source of information in changing proximal stimuli is the change in the change over time, i.e., the change in velocity of motion elements. Studies in kinetic depth perception have almost completely neglected proximal velocity change, but it should not be overlooked that this might provide additional information of depth.

Concerning circular motion in depth, Johansson (1958) reported that a point moving back and forth and changing velocity sinusoidally is frequently perceived as a circular motion in depth with more or less constant velocity. Correspondingly, other types of proximal velocity change may be perceived as translatory motion in depth. A translatory motion in depth with constant velocity is projected on the picture plane as a motion where the angular velocity is inversely related to the square of the viewing distance. The perceptually constant velocity of such a stimulus has been found to be accompanied by an impression of translatory motion in depth (Toshima, 1967). Evidently, there is a close relationship between perceived velocity constancy and impression of motion in depth.

The purpose of this paper is to study how and to what degree proximal velocity changes determine perceived translatory motion in depth. Changes in the distribution of light on a picture plane will here be used to describe the proximal changes. An earlier attack on this problem has been made by Börjesson and von Hofsten (1971), using motion patterns with stepwise changing velocity (the motion was divided into 10 subsections within each of which the velocity was constant). They found that great changes in the velocity of a proximal motion favor

\footnotetext{
*The author is indebted to Professor Gunnar Johansson and to Erik Börjesson for valuable discussions and for their comments on the manuscript. This investigation was made possible by grants to Professor Johansson from the Swedish Council for Social Science Research and the Tricentennial Fund of the Bank of Sweden.
}

perceived translatory motion in depth. In the present study, the problem will be more thoroughly examined, using motion patterns with continuously changing velocity. ${ }^{1}$ A preliminary experiment by the author, using continuously changing velocity patterns, indicated that certain conditions of proximal velocity change of a single dot gave stable percepts of motion in depth.

\section{Proximal Analysis}

A distal dot moving in depth along a straight path is represented on a picture plane by a motion with changing velocity toward or away from a perspective point at infinity at which the velocity of the projected motion would be zero. The distance between the projected dot and the perspective point is inversely related to the distance between the distal dot and the eye. The proximal velocity of the dot relative to its distance to the perspective point is equal to the velocity of the distal dot relative to its distance to the eye. In the following geometrical analysis of the proximal velocity change, however, absolute proximal and distal velocities are considered.

If the distal dot is receding with constant velocity (V), the covered proximal distance (d) at a certain time ( $t)$ can be expressed as:

$$
\mathrm{d}=\frac{\mathrm{K} \cdot \mathrm{R}}{\mathrm{D}_{0}}-\frac{\mathrm{K} \cdot \mathrm{R}}{\mathrm{D}_{0}+\mathrm{V} \cdot \mathrm{t}}
$$

where $\mathrm{K}$ is the distance from the eye to the picture plane, $D_{0}$ the initial depth distance to the dot (depth distances are here measured along the line of sight), and $\mathrm{R}$ the frontoparallel distance from the dot to the projection line parallel with the motion path. Using proximal stimuli, Eq. 1 is difficult to handle. It is easily rewritten as:

$$
d=\frac{d_{T} \cdot\left(D_{0}+V \cdot T\right) \cdot t}{\left(D_{0}+V \cdot t\right) \cdot T} ; 0 \leqslant t \leqslant T
$$




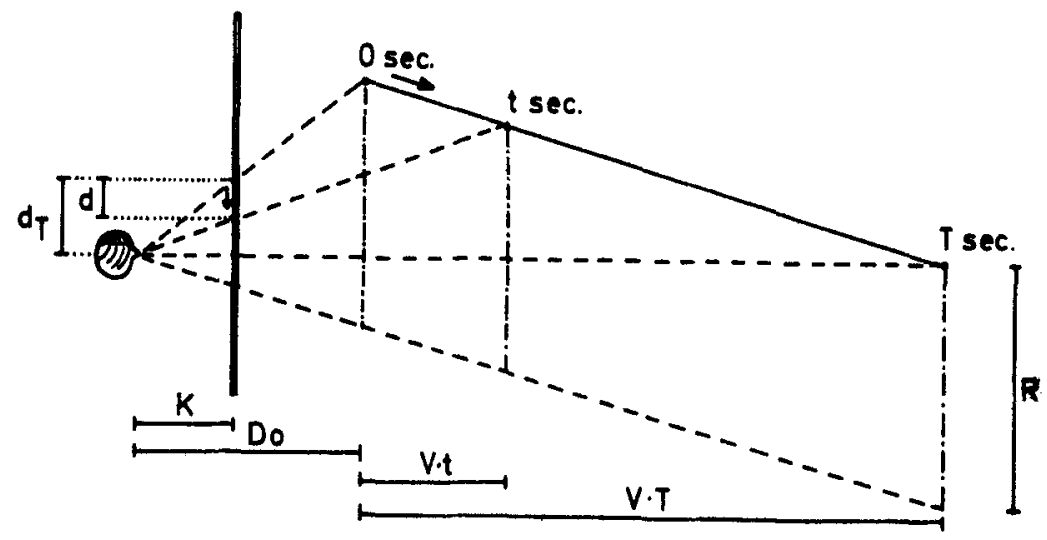

Fig. 1. Definition of symbols.

$$
P=V \cdot T / D_{0}
$$

where $T$ is the total duration of the motion and $d_{T}$ its total proximal extension. If all distal distances are measured relative to the initial depth distance to the dot $\left(D_{0}\right)$, then

$$
d=\frac{d_{T} \cdot(p+1) \cdot t}{(T+p \cdot t)}
$$

where $p=V T / D_{0}$, i.e., the relative amount of depth motion.

The proximal velocity (v) is obtained by differentiating Eq. 3 with respect to t:

$$
v=\frac{d_{T} \cdot(p+1) \cdot T}{(T+p \cdot t)^{2}} .
$$

The following can be said about different aspects of proximal velocity change:

(1) The amount of relative velocity change of the proximal motion is determined by the relative amount of distance in depth traveled $(p)$

$$
\mathrm{v}_{0} / \mathrm{v}_{\mathbf{T}}=(\mathrm{p}+1)^{2},
$$

where $v_{0}$ is the maximal proximal velocity $(t=0)$ and $v_{T}$ is the minimum proximal velocity $(\mathrm{t}=\mathrm{T})$.

(2) The relative rate of proximal velocity change $\left(a_{v}\right)$ is determined by the time $(t)$ and by the relative distal depth velocity $(\mathrm{p} / \mathrm{T})$.

$$
a_{v}=\frac{2 p / T}{t \cdot p / T+1},
$$

where $\mathrm{p}$ and $\mathrm{T}$ are defined as above.

(3) Finally, the absolute rate of proximal velocity change (a) is determined mainly by the relative distal depth velocity $(p / T)$ and the extension of the proximal motion $\left(d_{T}\right)$ as well as by the time variable $(t)$.

$$
a=\frac{2 d_{T} \cdot p / T(p / T+1 / T)}{(t \cdot p / T+1)^{3}},
$$

where $d_{T}, p$, and $T$ are defined as above.

\section{EXPERIMENT I}

In Experiment I, proximal velocity change was varied by using different relative amounts of simulated depth motion (p) and different exposuretimes (T). The extension of the proximal motion was constant. Experiment I had two purposes: (1) to test the hypothesis that a change in proximal velocity in accordance with Eq. 4 determines perceived translatory motion in depth; (2) to study what determines the strength of the depth impression, the amount of relative velocity change (Eq. 5) or the rate of change in velocity, relative or absolute (Eqs. 6 and 7). If the amount of relative velocity change is the critical factor, the exposure time should not affect the strength of the depth impression.

\section{Method}

Apparatus. A digital computer (Linc-8) was programmed to generate the motion patterns. The analog output of the computer was fed into an oscilloscope (Tectronix 565) from which the pattern was projected onto a translucent screen. In order to minimize cues of two-dimensionality from the screen, a collimator lens giving parallel light rays was placed between the screen and the $S$. This apparatus was used in all experiments.

Stimuli. The stimuli consisted of one dot moving back and forth in a horizontal path. When it was moving to the right, the velocity decreased according to Eq. 4 , and when it was moving to the lft, the velocity increased. The simulated depth motions of the velocity patterns in Experiment I are shown in Fig. 2.

One pattern with constant proximal velocity (Pattern A) and five patterns with changing proximal velocity (Patterns B-F) were included in Experiment I. Patterns B-F simulated distal dots receiding $1.25,2.5,3.33,5$, and 10 times the initial distance in depth, respectively. The change in proximal velocity over time for the different patterns is shown in Fig. 3.

The maximum proximal velocity of Patterns B-F was 4.76 , $12.25,19.75,36$, and 121 times greater than the minimum velocity, respec tively.

The velocity patterns were run under three conditions of total duration for each phase of motion: 2,4 , and $6 \mathrm{sec}$. At the turning points, the dot disappeared for $0.7 \mathrm{sec}$. Thus, a full cycle was completed in $5.4,9.4$, or $13.4 \mathrm{sec}$.

The extension of the proximal motion always subtended a visual angle of $14 \mathrm{deg}$. 


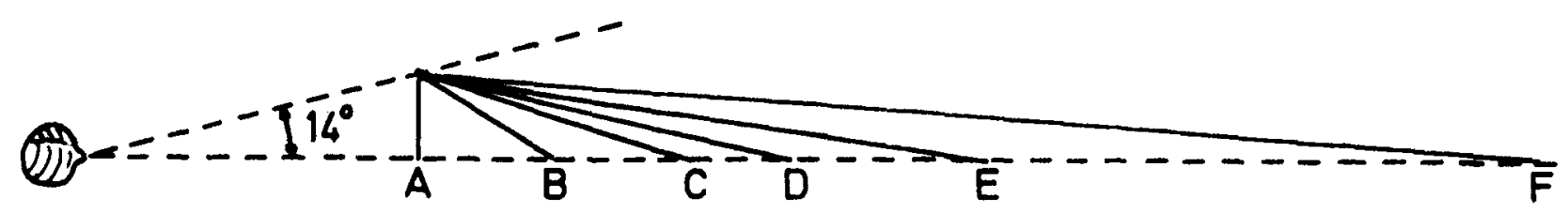

Fig. 2. The simulate;distal motions of the patterns used in Experiment I.

Viewing Conditions and Procedure. The $\mathrm{S}$ was seated about $20 \mathrm{~cm}$ in front of the collimator lens and was told that a moving dot would appear on the screen. The $S$ was asked to report whether he perceived the dot to move at approximately the same distance all the time or whether he perceived it to change its distance to him during the motion. When the latter alternative was reported, the $S$ was further asked to report at which part of the motion track he perceived the dot to be nearest to him and whether the impression of motion in depth was strong or weak. After two test trials, the different velocity patterns were presented once each in randomized order. During each presentation, the room was darkened and the velocity pattern appeared in a random phase of its cyclic course. The $S$ looked binocularly at the pattern until he was ready to give his report. Then the room was lighted and the report written down,

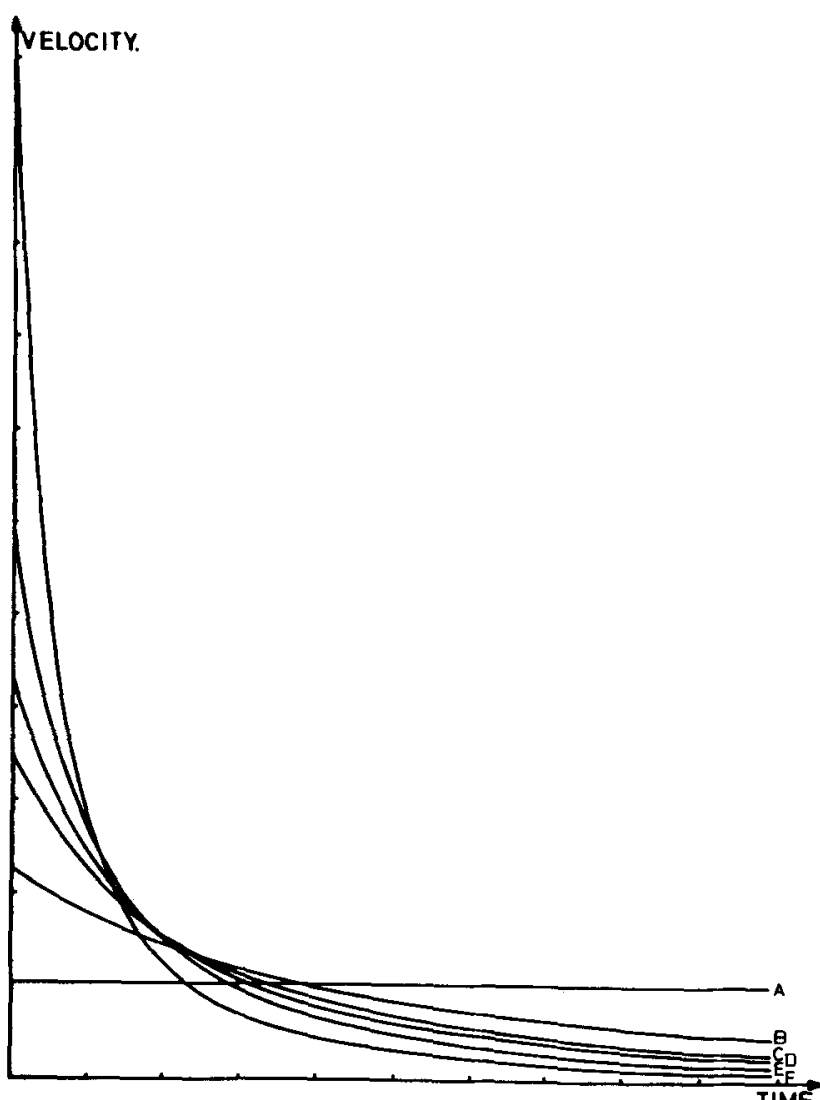

Fig. 3. The change in proximal velocity over time for the different patterns used in Experiment $I$. The three conditions of total exposure times $(2,4$, and 6 sec) corresponded to a constant velocity of Pattern A of $7 \mathrm{deg} / \mathrm{sec}, 3.5 \mathrm{deg} / \mathrm{sec}$, and $2.33 \mathrm{deg} / \mathrm{sec}$, respectively.
Table 1

Frequencies of Strong, Weak, and No Impression of Motion in Depth for the Different Conditions in Experiment $I$

\begin{tabular}{|c|c|c|c|c|c|c|c|}
\hline \multirow{2}{*}{$\begin{array}{l}\text { Dura- } \\
\text { tion }\end{array}$} & \multirow{2}{*}{$\begin{array}{c}\text { Depth } \\
\text { Impression }\end{array}$} & \multicolumn{6}{|c|}{ Velocity Patterns } \\
\hline & & A & B & $\mathrm{C}$ & D & $\mathrm{E}$ & $\mathbf{F}$ \\
\hline \multirow{3}{*}{$2 \mathrm{Sec}$} & Strong & 0 & 4 & 8 & 10 & 10 & 8 \\
\hline & Weak & 1 & 7 & 3 & 4 & 3 & 4 \\
\hline & No & 15 & 5 & 5 & 2 & 3 & 4 \\
\hline \multirow{3}{*}{$4 \mathrm{Sec}$} & Strong & 0 & 3 & 5 & 9 & 9 & 13 \\
\hline & Weak & 2 & 4 & 5 & 3 & 5 & 2 \\
\hline & No & 14 & 9 & 6 & 4 & 2 & 1 \\
\hline \multirow{3}{*}{$6 \mathrm{Sec}$} & Strong & 0 & 1 & 7 & 5 & 10 & 6 \\
\hline & Weak & 0 & 4 & 3 & 6 & 3 & 8 \\
\hline & No & 16 & 11 & 6 & 5 & 3 & 2 \\
\hline
\end{tabular}

whereaf ter the next pattern was presented.

Subjects. Eighteen Ss participated in Experiment I.

\section{Results}

When motion in depth was reported, the dot was always perceived to be nearest to the $S$ when having its maximal velocity.

Two Ss always perceived the dots as moving in the fronto-parallel plane with changing velocity. These Ss were excluded from the data treatment. The reports of the other 16 Ss were classified into three categories: strong, weak, or no impression of motion in depth. The frequencies of these categories are shown in Table 1.

Table 1 shows that the patterns with changing velocity (Patterns B-F) evoked more percepts of motion in depth than Pattern A, having constant velocity. This effect was tested with Wilcoxon matched-pairs signed-ranks test (Siegel, 1956). It was found that each of Patterns B-F gave significantly more percepts of motion in depth than Pattern $A(p<.01$, one-tailed test).

The relative amount of simulated depth motion (p) had a systematic effect on perceived motion in depth. The greater the simulated depth motion, the greater the number of reported motions in depth. Patterns $\mathrm{E}$ and $\mathrm{F}$ almost always evoked perceived depth motion. The effect of relative amount of simulated depth motion (p) on perceived motion in depth was tested with the Friedman two-way analysis of variance (Siegel, 1956). The obtained value of $\chi^{2}=23.4$ is significant at a level of .001 for $\mathrm{df}=4$.

Time to complete the change (T) did affect the 


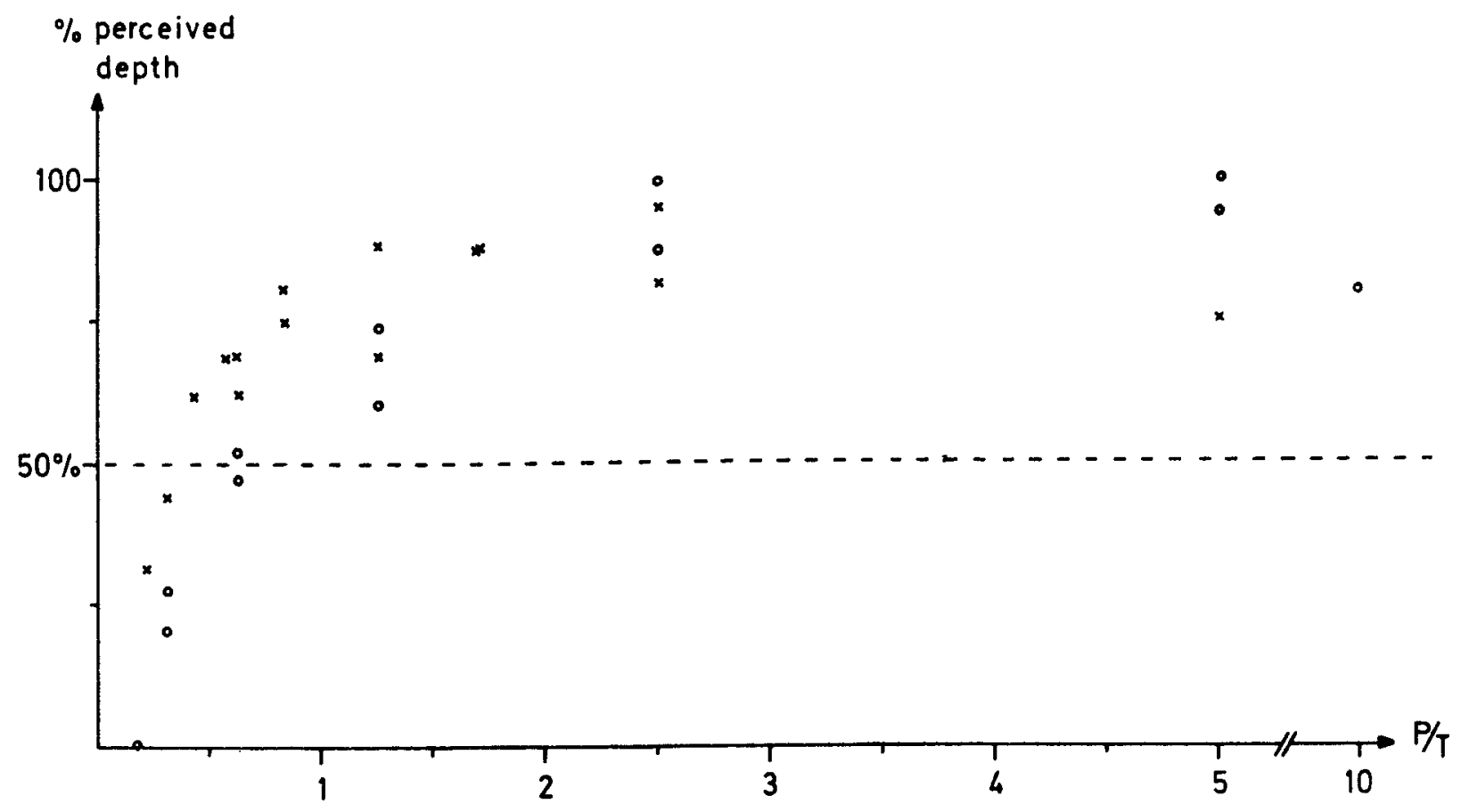

Fig. 4. Percent reports of motion in depth under the different conditions of simulated relative distal depth velocity (p/T) for Experiment I (crosses) and Experiment 11 (circles).

strength of the depth impression. Patterns B, C, and D evoked more percepts of motion in depth when the exposure time was short, Pattern $\mathrm{E}$ was not affected by this variable, and Pattern $F$ evoked slightly more motions in depth for the longer exposures. Spontaneous reports indicated that the perceived motion path was usually bent.

\section{Discussion and Conclusions}

To perceive a single dot moving in depth, there must be a change in the angular velocity of the proximal motion. If the proximal velocity is constant, no motion in depth is perceived, only motion in a frontoparallel plane. Experiment I clearly shows that, within certain limits, changes in the proximal velocity in accordance with Eq. 4 are utilized by the visual system to determine perceived translatory motion in depth. The influence of relative amount of simulated depth motion (p) and exposure time (T) on the perception of the patterns in Experiment $I$ indicates that these limits are primarily a function, not of the amount of proximal relative velocity change, but of the rate of proximal velocity change. The effect of time (T) should, however, be further tested. In Fig. 4, the percent reports of motion in depth for each stimulus used in Experiment $I$ is plotted against their value of simulated relative depth velocity $(\mathrm{p} / \mathrm{T})$. Figure 4 shows that there is a fairly good agreement in the tendency to perceive depth motion for dots having similar values of $\mathrm{p} / \mathrm{T}$, but different values of $\mathrm{p}$ and $\mathrm{T}$.

\section{EXPERIMENT II}

Experiment I indicated that the rate of change in proximal velocity determines the strength of the depth impression. The rate of change in proximal velocity is dependent on the value of $p / T$, i.e., simulated relative depth velocity. If the rate of change in proximal velocity is the critical variable, then the perception of motion in depth should be independent of whether the stimuli are based upon varying $p$ or $T$. In the previous experiment, the stimuli were based mainly upon varying $p$. The first purpose of Experiment II was to further test the hypothesis that the rate of change in proximal velocity determines the strength of the depth impression. This was done by mainly varying the exposure time (T). The second purpose was to study more closely the form of the perceived motion path.

\section{Method}

Stimuli. The stimulus patterns used were identical with Patterns $\mathrm{C}$ and $\mathrm{E}$ in Experiment I, simulating distal dots receding 2.5 and 5 times the initial distance in depth, respectively. The two patterns were run under six conditions of total exposure time, each phase of motion lasting for $0.5,1,2,4,8$, or $16 \mathrm{sec}$. At the turning points, the dot disappeared for $0.7 \mathrm{sec}$. Thus, a full cycle was completed in $2.4,3.4,5.4,9.4,17.4$, or $33.4 \mathrm{sec}$. The extension of the proximal motion always subtended a visual angle of $13 \mathrm{deg}$.

Viewing Conditions and Procedure. These were the same as in Experiment I, except that the perceived form of the motion track was also asked for when motion in depth was reported.

Subjects. Fifteen Ss participated in Fxperiment II. 


\section{Results}

When motion in depth was reported, the dot was always perceived to be nearest to the $S$ when having its maximal velocity.

The reports were classified into the same categories as in Experiment $I$. The frequencies of these categories are presented in Table 2.

The time to complete the change $(T)$ had a systematic effect on perceived motion in depth. This effect was tested with the Friedman two-way analysis of variance (Siegel, 1956). The obtained value of $\chi^{2}=35.5$ is significant at a level of .001 for $\mathrm{df}=5$.

In Fig. 4, the percent reports of motion in depth for the stimuli used in Experiment II are plotted against their value of simulated relative depth velocity $(\mathrm{p} / \mathrm{T})$. Figure 4 shows that there is good agreement between Experiments I and II. The relation between simulated relative depth velocity and the tendency to perceive motion in depth remains the same independently of whether the stimuli are based upon varying $p$ or $T$. The tendency to perceive depth arises from no depth percepts at the lowest rate of change in proximal velocity $(\mathrm{p} / \mathrm{T}=0.16)$ to almost unambiguously perceived depth motion at a rate of change in proximal velocity corresponding to a value of $\mathrm{p} / \mathrm{T}$ of about 1.0 , i.e., a simulated velocity in depth equal to the initial distance per second. At very high rates of change in proximal velocity, the strength of the depth impression is slightly diminished, which is reflected in the number of strong percepts of motion in depth, though not evident from the total number of depth percepts.

As reported by some Ss in Experiment I, the motion path was in most cases not perceived as straight. When receding, the dot was usually perceived to move along a path which was bent toward the $S$, as in Fig. 5A. This effect was particularly marked for patterns with high rates of change in proximal velocity. When approaching, the dot was usually perceived to move along a path that was bent away from the Ss, as in Fig. 5B. This effect was particularly marked for patterns with low rates of proximal velocity change.

The following spontaneous remarks regarding the perceived change in velocity of the dot were reported by several Ss. When the depth impression was impaired, i.e., the angle between the perceived motion track and the frontoparallel plane was underestimated, there was a

Table 2

Frequencies of Strong, Weak, and No Impression of Motion in Depth for the Different Conditions in Experiment II

\begin{tabular}{llrrrrrr}
\hline & & \multicolumn{6}{c}{ Exposure Times (Sec) } \\
\cline { 3 - 7 } & $\begin{array}{c}\text { Depth } \\
\text { Impression }\end{array}$ & 0.5 & 1 & 2 & 4 & 8 & 16 \\
\hline \multirow{3}{*}{ Pattern E } & Strong & 5 & 8 & 10 & 6 & 3 & 2 \\
& Weak & 7 & 7 & 5 & 3 & 4 & 2 \\
& No & 3 & 0 & 0 & 6 & 8 & 11 \\
& Strong & 6 & 6 & 7 & 4 & 0 & 0 \\
Pattern C & Weak & 8 & 7 & 4 & 4 & 3 & 0 \\
& No & 1 & 2 & 4 & 7 & 12 & 15 \\
\hline
\end{tabular}
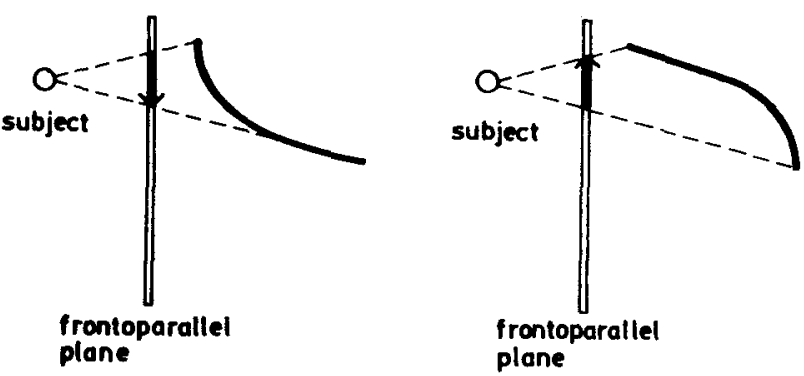

Fig. 5. (A) The perceived motion track of a dot with diminishing proximal velocity. (B) The perceived motion track of a dot with increasing proximal velocity.

pronounced change in the perceived velocity of the dot. Only when the motion path was perceived as approximately straight was the velocity of the dot perceived as more or less constant.

\section{Conclusions}

It is concluded from Experiments I and II that the perception of translatory motion in depth induced by a proximal velocity change in accordance with Eq. 4 is determined by the rate of change in the proximal velocity. The number of percepts of motion in depth for the different rates of change in proximal velocity indicates that the rate of change must be above some threshold value. The result also indicates that when the rate of change in proximal velocity is very high, the strength of the depth impression is somewhat diminished. The perceived form of the motion track further supports this conclusion. At very high and very low rates of change in proximal velocity, the depth impression was diminished, i.e., the angle between the perceived motion track and the frontoparallel plane was small. This is in accordance with findings concerning spatial changes. Metzger (1934) found that the impression of depth is diminished when the rate of spatial change is above or below certain values.

Concerning the perceived form of the motion track, it should be noted that the depth impression was impaired mostly for very high and very low rates of change in velocity occurring in the beginning of the motion (see Fig. 5). This result indicates that the most critical phase in the perceptual process is the initial formation of the depth percept. Once the percept of motion in depth is formed, much greater variations in the rate of change in velocity is tolerated with preserved impression of motion in depth. There may also be a general short delay in the formation of the percept. In studies of frontoparallel velocity perception, Gottsdanker et al (1961) and Runeson (1973) found that the very first part of the motion was difficult to perceive.

\section{EXPERIMENT III}

The previous experiments have shown that the rate of change in proximal velocity determines the perception 
Table 3

Frequencies of Strong, Weak, and No Impression of Motion in Depth for the Different Conditions in Experiment III

\begin{tabular}{|c|c|c|c|c|c|}
\hline \multirow{2}{*}{$\begin{array}{c}\text { Exten- } \\
\text { sion }\end{array}$} & \multirow{2}{*}{$\begin{array}{c}\text { Depth } \\
\text { Impression }\end{array}$} & \multicolumn{2}{|c|}{ Pattern F } & \multicolumn{2}{|c|}{ Pattern C } \\
\hline & & $2 \mathrm{Sec}$ & $4 \mathrm{Sec}$ & $2 \mathrm{Sec}$ & $4 \mathrm{Sec}$ \\
\hline \multirow{3}{*}{14 Deg } & Strong & 10 & 10 & 5 & 2 \\
\hline & Weak & 4 & 2 & 7 & 7 \\
\hline & No & 1 & 3 & 3 & 6 \\
\hline \multirow{3}{*}{$7 \mathrm{Deg}$} & Strong & 5 & 5 & 1 & 1 \\
\hline & Weak & 8 & 4 & 7 & 6 \\
\hline & No & 2 & 6 & 7 & 8 \\
\hline \multirow{3}{*}{ 3.5 Deg } & Strong & 1 & 0 & 1 & 1 \\
\hline & Weak & 7 & 5 & 6 & 2 \\
\hline & No & 7 & 10 & 8 & 12 \\
\hline
\end{tabular}

of translatory motion in depth. In Experiment III, the question is asked whether the critical aspect is the absolute (Eq. 7) or the relative (Eq. 6) rate of change in velocity. To test this, the extension of the proximal motion was varied. This affects the absolute, but not the relative, rate of change in velocity.

\section{Method}

Stimuli. The stimulus patterns were identical to Patterns C and $F$ in Experiment $I$, simulating distal dots receding 2.5 and 10 times the initial distance in depth, respectively. The patterns were run under two conditions of total exposure time, 2 and $4 \mathrm{sec}$ for one phase of motion. The values of $\mathrm{p} / \mathrm{T}$, i.e., simulated relative depth velocity, for the conditions of relative rate of proximal velocity change were thus $5,2.5,1.25$, and 0.625 . The extension of the proximal motion path was $3.5,7$, or $14 \mathrm{deg}$.

Viewing Conditions and Procedure. These were the same as in Experiment I.

Subjects. Fifteen Ss participated in Experiment III.

\section{Results}

When motion in depth was reported, the dot was always perceived to be nearest to the $S$ when having its maximal velocity.

The reports were classified into the same categories as in Experiment I. The frequencies of these categories are represented in Table 3. Table 3 shows that the influence of $p / T$ on the perception of translatory motion in depth is in accordance with the previous experiments for the greatest extension of the proximal motion path $(14 \mathrm{deg})$. There is a clear effect of the extension of the proximal motion path on the amount of perceived motion in depth, the motion paths of $3.5,7$, and 14 deg yielding a total of 23,37 , and 47 percepts of motion in depth, respectively. These data were used in testing the effect of extension of the proximal motion path with the Friedman two-way analysis of variance (Siegel, 1956). The obtained value of $\chi^{2}=12.5$ is significant at a level of .01 for $\mathrm{df}=2$.

\section{Conclusions}

It isconcluded from Experiment III that the critical factor of proximal velocity change determining the thresholds of perceived translatory motion in depth is the absolute rate of change in velocity.

\section{DISCUSSION}

Phenomenological reports were used as a measure of the perceived characteristics of the motion patterns. Their reliability is supported by the following facts. First, the effects of the physical variables manipulated on perceived motion in depth are highly significant, and the results of the three experiments support each other. Secondly, in the cases of perceived motion in depth, the dot was consistently perceived to recede when the velocity decreased and to approach when the velocity increased. There was also a high agreement between the Ss concerning the perceived form of the motion track.

The results clearly show that changes in proximal velocity corresponding to distal depth motions with constant speed, within certain limits, evoke stable percepts of motion in depth. The limits were found to be determined by the absolute rate of change in proximal velocity. The number of reported depth motions, the reported strength of the percepts, and the reported form of the motion tracks all showed that the absolute rate of proximal velocity change must be above some threshold value if it is to be utilized by the visual system. It was also found that the strength of the depth impression is somewhat impaired if the absolute rate of change is too great. The absolute rate of proximal velocity change depends on the distal depth velocity of the dot, relative to its distance to the eye, and on the extension of the projected motion. Consequently, the perception of translatory motion in depth depends on the value of both these factors. If the extension of the projected motion is small, the relative distal velocity must be great if motion in depth is to be perceived, and vice versa.

The absolute values of the thresholds obtained in this investigation should not be taken too seriously. Motion thresholds are much greater in homogeneous fields than when reference objects are present (Aubert, 1886; Koffka, 1935). Therefore, there is reason to believe that the thresholds related to perceived motion in depth of a dot with changing velocity will also be much lower when other objects are presented in the field.

Although all stimuli simulated depth motions along straight paths, the perceived motion track was usually bent (Fig. 5). When the depth impression was impaired, i.e., the angle between the perceived motion track and the frontoparallel plane was underestimated, spontaneous reports indicated that there was a pronounced change in the perceived velocity of the dot. This finding, which shows that proximal velocity change may be partly perceived as motion in depth and partly as a velocity change of the dot, is consistent with findings concerning spatial changes (Marmolin, 1973). The problem of how perceived velocity constancy comes about cannot be handled in the present context by the introduction of assumed constant velocity of the dot.Rather, the perceptual system seems to work in a very mechanical and automatic way. There seems to be 
an invariant relation between perceived velocity change and perceived motion in depth. The more of the proximal velocity change that is perceived as motion in depth, the less is left over to evoke perceived velocity change of the dot.

The perceived form of the motion track indicates that the most critical phase in the perceptual process is the initial formation of the depth percept. The limits for perceiving motion in depth found in this investigation are with necessity related to this aspect of the perceptual process. Once the percept is formed, much greater variations in the rate of change in proximal velocity are tolerated with preserved impression of motion in depth.

It can be concluded from the present investigation that temporal proximal factors related to spatial changes as well as to velocity changes are utilized by the visual system to determine space perception. In everyday life, these two temporal factors supplement each other in the perception of rigid objects with constant velocities. It is, therefore, important that both spatial changes and velocity changes are considered in the study of kinetic depth perception.

\section{REFERENCES}

Aubert, H. Die Bewegungsemp findung. Archiv für die Gesamte Physiologie, 1886, 39, 347-370.

Börjesson, E., \& v. Hofsten, C. Determinants of depth perception in motion patterns. Report No. 101, Department of Psychology. University of Uppsala, 1971.
Börjesson, E., \& v. Hofsten, C. Spatial determinants of depth perception in two-dot motion patterns. Perception \& Psychophy sics, 1972, 11, 263-268.

Börjesson, E., \& v. Hofsten, C. Visual perception of motion in depth: Application of a vector model to three-dot motion patterns. Perception \& Psychophysics, 1973, 13, 169-179.

Gottsdanker, R., Frick, J. W.. \& Lockard, R. B. Identifying the acceleration of visual targets. British Journal of Psychology, $1961,52,31-42$.

Johansson, G. Rigidity, stability and motion in perceptual space. Acta Psychologiea, 1958, 14, 359-370.

Johansson, G. Perception of motion and changing form. Scandinavian Journal of Psychology, 1964, 5, 181-208.

Koffka, K. Principles of Gestalt psychology. New York: Harcourt, Brace, 1935.

Marmolin, $H$. Visually perceived motion in depth resulting from proximal changes. I. Perception \& Psychophysics, 1973, 14, 133-142.

Metzger, W. Tiefen Erscheinungen in Optischen Bewegungsfeldern. Psychologische Forschung, 1934, 20, 195-260.

Runeson, S. Constant velocity-not perceived as such. Report No. 140, Department of Psychology, University of Uppsala, 1973.

Siegel, S. Nonparametric statistics for the behavioral sciences. New York: McGraw-Hill, 1956.

Toshima, Y. Experimental studies on radial velocity constancy. In Y. Akishige (Ed.), Experimental researches on the structure of the perceptual space. VI. Bulletin of the Faculty of Literature of Kyushu University, Fukuoka, Japan, 1967, 11, 1-37.

Wallach, H., \& O'Connell, D. N. The kinetic depth effect. Journal of Experimental Psychology, 1953, 45, 205-217.

\section{NOTE}

1. This function was approximated by sampling the distance traveled along the projection surface in a small interval of time. Each motion consisted of 300 such samplings.

(Received for publication June 5, 1973 revision received December 14, 1973.) 